\author{
Jean-Michel Jeannin
}

\section{Einleitung}

Citrus medica (dt. Zitronatszitrone, fr. Cédrat, eng. Citron) ist ein immergrüner, 2,4-4,5 $\mathrm{m}$ hoher Strauch oder Baum aus der Familie der Rautengewächse. Die ledrigen Blätter sind oval-lanzettlich oder oval-eliptisch, 6-18 cm lang und duften nach Zitrone. In den Blattachseln befinden sich Dornen. Die rund $4 \mathrm{~cm}$ grossen Blüten sind weiss und bisweilen an der Aussenseite rosa oder purpurfarben. Sie zählen 4-5 Kronblätter und 50-60 Staubfäden. Die im reifen Zustand orangegelbe Frucht kann zwischen 9 und $30 \mathrm{~cm}$ gross werden. Oberfläche und Gestalt der Frucht sind sehr variabel. Die Aussenseite ist häufig unregelmässig gefurcht und gebuckelt. Die Schale ist sehr dick und sehr aromatisch. Die gelbliche, rosafarbe-

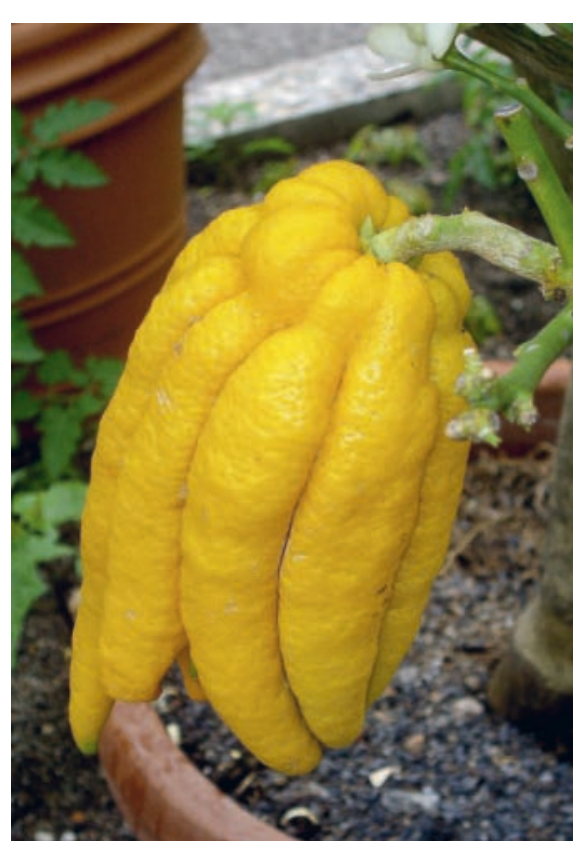

Abb. 1. Frucht von C. medica var. sarcodactylis («Buddhas Hand»). Botanischer Garten Basel, 2011.

\title{
Exotische Heilpflanzen (11)
}

\section{Citrus medica L. (Rutaceae); Citrus medica var. sarcodactylis Hort. (Rutaceae)}

ne oder grünliche Pulpa ist in 14-15 Segmente aufgeteilt [1]. C. medica var. sarcodactylis (Abb. 1) unterscheidet sich von $C$. medica hauptsächlich durch die skurrile Form der Früchte: Diese laufen an dem Stiel entgegengesetzten Ende in fingerförmige Wulste aus, die sich bisweilen aufbiegen [1].

\section{Verwendung}

Die in kleine Stücke geschnittene und kandierte Schale von C. medica (Zitronat) wird als Kuchengewürz verwendet. Das Fruchtfleisch kann als Konfitüre Verwendung finden [2]. Destillate der Schale, Blüten, Blätter und unverholzten Zweige werden in der Parfümindustrie eingesetzt [1].

\section{Traditionelle Verwendung}

Die frische Schale von C. medica eignet sich als appetitanregendes und verdauungsförderndes Mittel [2]. Das ätherische Öl der Schale gilt als starkes Antiseptikum [3]. In Indien gilt die Schale als Mittel zur Behandlung der Dysenterie (Ruhr) und wird zur Bekämpfung von schlechtem Mundgeruch gegessen. Ein Destillat der Schale wird als Sedativum gegeben. Die kandierte Schale wird in China als Stomachicum, Stimulans, Tonikum und Expektorans verabreicht. In Südostasien werden die Kerne als Wurmmittel gegeben. In Panama wird das ätherische Öl der Schale als Antibiotikum betrachtet [1].

\section{Phytochemie}

Die frische Schale enthält Limonen $(60-80 \%), \quad \gamma$-Terpinen $(5-32 \%$ in
C. medica var. sarcodactylis), $\beta$-Pinen und Citral [2], ferner Hesperoside, Naringoside, Ecryoside, Ascorbinsäure, Hesperidin und Rutin. Die Blätter enthalten 28\% Erucylamide, 18\% Limonen und 13\% Citral [4].

\section{Aktuelle Forschung}

Sood et al. [5] haben im Tiermodell die analgetische und antiphlogistische Wirkung verschiedener Extrakte der Schale von C. medica untersucht. Die Autoren kamen zu dem Schluss, dass bestimmte Extrakte in vitro eine hohe antioxidative und in vivo eine nachweisbare analgetische und antiphlogistische Wirkung besitzen. Diese Wirkungen könnten von Flavonoiden und Phenolsäuren stammen. Im Tierversuch konnte eine hypoglykämische und antidiabetische Wirkung mit der peroralen Verabreichung einer Zubereitung aus der ganzen Frucht von C. medica var. sarcodactylis erzielt werden [6].

\section{Literatur}

1 Meena AK, et al: A review on citron - pharmacognosy, phytochemistry and medical uses. Inter Res J Pharmacy 2011;2:14-19.

2 Teuscher E: Gewürzdrogen. Stuttgart, Wissenschaftliche Verlagsgesellschaft, 2003, pp 107109.

3 Lemoine E: Les épices de Santé. Paris, Editions Molière, 2002, p 554.

4 Bhuiyan MNI, et al: Constituents of peel and leaf essential oils of Citrus medica. J Scient Res 2009;1:387-392.

5 Sood S, et al: Therapeutic potential of Citrus medica L. peel extract in carrageenan induced inflammatory pain in rat. Res J Med Plant 2009;3:123-133.

-6 Peng $\mathrm{CH}$, et al: Insulin secretagogue bioactivity of finger citron fruit (Citrus medica L. var. sarcodactylis Hort, Rutaceae). J Agric Food Chem 2009;57:8812-8819.

\section{KARGER}

Fax +497614520714 Information@Karger.d www.karger.com (c) 2011 S. Karger GmbH, Freiburg
Dipl. med. biol. Jean-Michel Jeannin Holeestrasse 43, 4054 Basel, Schweiz

Tel. +41 61-4215991, Fax - 4230313

jmjeannin@datanetworks.ch 